\title{
On James and Jordan-von Neumann constants and the normal structure coefficient of Banach spaces
}

\author{
by \\ Mikio Kato (Kitakyushu), LeCH MaligRAnda (Luleå), and \\ YASUJi TAKAHASHI (Soja)
}

\begin{abstract}
Some relations between the James (or non-square) constant $J(X)$ and the Jordan-von Neumann constant $C_{\mathrm{NJ}}(X)$, and the normal structure coefficient $N(X)$ of Banach spaces $X$ are investigated. Relations between $J(X)$ and $J\left(X^{*}\right)$ are given as an answer to a problem of Gao and Lau [16]. Connections between $C_{\mathrm{NJ}}(X)$ and $J(X)$ are also shown. The normal structure coefficient of a Banach space is estimated by the $C_{\mathrm{NJ}}(X)$-constant, which implies that a Banach space with $C_{\mathrm{NJ}}(X)$-constant less than $5 / 4$ has the fixed point property.
\end{abstract}

0. Introduction. Several results on the Jordan-von Neumann constant (which is usually called the von Neumann-Jordan constant) of a Banach space $X$, denoted by $C_{\mathrm{NJ}}(X)$, have recently been obtained by the authors [25], [26], [38] (we also refer to [23] and [8] for the classical results). In particular, they determined or estimated $C_{\mathrm{NJ}}(X)$ for various spaces $X$, and showed that some properties of $X$ such as uniform non-squareness, superreflexivity or type and cotype can be described in terms of the constant $C_{\mathrm{NJ}}(X)$.

The aim of this paper is to clarify some relations beween the $C_{\mathrm{NJ}}(X)$ constant and some other geometrical constants, especially the non-square constant $J(X)$ of James and the normal structure coefficient $N(X)$. In addition, we investigate the James constant $J(X)$ more carefully. Everything is supported by several examples of concrete Banach spaces with the calculation of these constants.

The paper is organized as follows: In Section 1 we collect necessary properties of the modulus of convexity and modulus of smoothness. In Section 2 the uniformly non-square spaces and the James constant $J(X)$ are consid-

2000 Mathematics Subject Classification: 46B20, 46E30, 46A45, $46 \mathrm{~B} 25$.

Key words and phrases: uniformly convex spaces, uniformly smooth spaces, uniformly non-square spaces, James constant, Jordan-von Neumann constant, normal structure coefficient, fixed point property, Banach-Mazur distance. 
ered. A relation between $J(X)$ and $J\left(X^{*}\right)$ ( $X^{*}$ is the dual space of $X$ ) is given in Theorem 1, which partly answers a question of Gao and Lau [16], Question 7.3. Note that $J(X) \neq J\left(X^{*}\right)$ in general (cf. our Example 2). As a corollary we obtain the well known result that $X$ is uniformly non-square if and only if $X^{*}$ is uniformly non-square. Section 3 contains information about the $C_{\mathrm{NJ}}(X)$-constant and its connection with the $J(X)$-constant. The estimates of the $C_{\mathrm{NJ}}(X)$-constant from below and above by the $J(X)$-constant are the main result of this part (Theorem 3). We also calculate $J(X)$ and $C_{\mathrm{NJ}}(X)$ for $X$ being the two-dimensional Lorentz sequence space $\ell_{p, 2}$ with $p \geq 2$.

In Section 4 we consider the relation between the $C_{\mathrm{NJ}}(X)$-constant and the normal structure coefficient of $X$ in the context of the fixed point property. Gao and Lau [16] showed that if $J(X)<3 / 2$, then $X$ has normal structure. Prus [36] even estimated the normal structure coefficient $N(X)$ by $J(X)$. We present an estimate of $N(X)$ by the $C_{\mathrm{NJ}}(X)$-constant, which yields that if $C_{\mathrm{NJ}}(X)<5 / 4$, then the Banach space $X$ as well as its dual $X^{*}$ have the fixed point property. Finally, in the last Section 5, we compare the constants $J(X), C_{\mathrm{NJ}}(X)$ and $N(X)$ for $X$ and for an isomorphic space $Y$ by using the Banach-Mazur distance $d(X, Y)$. These estimates give the possibility to compute such constants for a Banach space with new but equivalent norms. Of course, these constants are not stable with respect to an equivalent renorming. We finish this part with the estimates of $J(X), C_{\mathrm{NJ}}(X)$ and $N(X)$ for the interesting Day-James $\ell_{p}-\ell_{q}$ spaces and the Bynum $b_{p, q}$ spaces. Some open problems are also posed.

1. Preliminaries. Let $X=(X,\|\cdot\|)$ be a real Banach space. The nontrivial Banach space will mean later on that $X$ is a real space and $\operatorname{dim} X \geq 2$ (but it can also be that $X$ is a complex space and $\operatorname{dim} X \geq 1$ ). Geometrical properties of a Banach space $X$ are determined by its unit ball $B_{X}=\{x \in$ $X:\|x\| \leq 1\}$ or its unit sphere $S_{X}=\{x \in X:\|x\|=1\}$. The modulus of convexity of $X$ (cf. [30], [34], [13], [35]) is a function $\delta_{X}:[0,2] \rightarrow[0,1]$ defined by

$$
\delta_{X}(\varepsilon)=\inf \left\{1-\|x+y\| / 2: x, y \in S_{X},\|x-y\| \geq \varepsilon\right\} .
$$

The function $\delta_{X}$ is continuous on $[0,2)$, increasing on $[0,2]$, strictly increasing on $\left[\varepsilon_{0}, 2\right]$, where $\varepsilon_{0}=\varepsilon_{0}(X)=\sup \left\{\varepsilon \in(0,2]: \delta_{X}(\varepsilon)=0\right\}$ is the so-called coefficient of convexity of $X$, and $\delta_{X}(\varepsilon) \leq \varepsilon / 2$ or even $\delta_{X}(\varepsilon) \leq \delta_{\ell_{2}}(\varepsilon)=1-\sqrt{1-\varepsilon^{2} / 4}$. Also $\lim _{\varepsilon \rightarrow 2^{-}} \delta_{X}(\varepsilon)=1-\varepsilon_{0}(X) / 2$. The function $\delta_{X}(\varepsilon) / \varepsilon$ is increasing on $(0,2]$ but $\delta_{X}(\varepsilon)$ is not necessarily convex (Liokumovich 1973; cf. also our easier Example 2). The space $X$ is called uniformly convex if $\delta_{X}(\varepsilon)>0$ for all $0<\varepsilon<2$, i.e., $\varepsilon_{0}(X)=0$. Day [9] (see also [30], [34]) proved the important result that if $X$ is non-trivial, then 


$$
\begin{aligned}
\delta_{X}(\varepsilon) & =\inf \left\{1-\|x+y\| / 2: x, y \in B_{X},\|x-y\| \geq \varepsilon\right\} \\
& =\inf \left\{1-\|x+y\| / 2: x, y \in S_{X},\|x-y\|=\varepsilon\right\} \\
& =\inf \left\{1-\|x+y\| / 2: x, y \in B_{X},\|x-y\|=\varepsilon\right\} .
\end{aligned}
$$

The modulus of smoothness of $X$ (cf. [9], [30], [34], [28]) is a function $\varrho_{X}$ : $[0, \infty) \rightarrow[0, \infty)$ defined by

$$
\varrho_{X}(\tau)=\sup \left\{\frac{\|x+\tau y\|+\|x-\tau y\|}{2}-1: x, y \in S_{X}\right\} .
$$

The function $\varrho_{X}$ is increasing, continuous, convex on $[0, \infty), \varrho_{X}(0)=0$ and $\varrho_{X}(\tau) \leq \tau$. Moreover, if $X$ is non-trivial, then $\varrho_{X}(\tau) \geq \varrho_{\ell_{2}}(\tau)=\sqrt{1+\tau^{2}}-1$ and the supremum in (1.3) can be taken over all $x, y \in B_{X}$. The space $X$ is called uniformly smooth if $\varrho_{X}^{\prime}(0)=\lim _{\tau \rightarrow 0} \varrho_{X}(\tau) / \tau=0$. The following remarkable duality relationship with the modulus of convexity is due to Lindenstrauss [28] (see also [30], [34]): for $\tau>0$,

$$
\begin{aligned}
\varrho_{X^{*}}(\tau) & =\sup \left\{\tau \varepsilon / 2-\delta_{X}(\varepsilon): \varepsilon \in[0,2]\right\}, \\
\varrho_{X}(\tau) & =\sup \left\{\tau \varepsilon / 2-\delta_{X^{*}}(\varepsilon): \varepsilon \in[0,2]\right\},
\end{aligned}
$$

which yields the equality $\varrho_{X}(\tau)=\varrho_{X * *}(\tau)$ and also the fact that $X$ is uniformly convex (resp. uniformly smooth) if and only if its dual $X^{*}$ is uniformly smooth (resp. uniformly convex). Moreover, if $X$ is either uniformly convex or uniformly smooth, then $X$ is reflexive.

REMARK 1. In the real one-dimensional case, i.e., when $X=(\mathbb{R},|\cdot|)$, the modulus of convexity $\delta_{X}(\varepsilon)$ from $(1.1)$ is $\delta_{X}(\varepsilon)=1$ for $0<\varepsilon \leq 2$, and the first one from (1.2) is $\delta_{X}(\varepsilon)=\varepsilon / 2$ for $0 \leq \varepsilon \leq 2$; the modulus of smoothness $\varrho_{X}(\tau)$ from (1.3) is in this case $\varrho_{X}(\tau)=\max \{0, \tau-1\}$ for $\tau \geq 0$.

2. Uniformly non-square spaces and James constant. A Banach space $X$ is called uniformly non-square (James, 1964) if there exists a $\delta \in$ $(0,1)$ such that for any $x, y \in S_{X}$ either $\|x+y\| / 2 \leq 1-\delta$ or $\|x-y\| / 2 \leq 1-\delta$. The constant

$$
J(X)=\sup \left\{\min (\|x+y\|,\|x-y\|): x, y \in S_{X}\right\}
$$

is called the non-square or James constant of $X$.

Proposition 1. Let $X$ be a non-trivial Banach space. The following conditions are equivalent.

(i) $X$ is uniformly non-square.

(ii) $\delta_{X}(\varepsilon)>0$ for some $0<\varepsilon<2$.

(iii) $\varepsilon_{0}(X)<2$.

(iv) $J(X)<2$.

(v) $\varrho_{X}\left(\tau_{0}\right)<\tau_{0}$ for some $\tau_{0}>0$.

(vi) $\varrho_{X}(\tau)<\tau$ for all $\tau>0$. 
(vii) $\varrho_{X}^{\prime}(0)=\lim _{\tau \rightarrow 0} \varrho_{X}(\tau) / \tau<1$.

(viii) $\varepsilon_{0}\left(X^{*}\right)<2$.

(ix) $\varrho_{X^{*}}^{\prime}(0)=\lim _{\tau \rightarrow 0} \varrho_{X^{*}}(\tau) / \tau<1$.

Proof. We have the equivalences:

$X$ is uniformly non-square

$$
\begin{aligned}
& \Leftrightarrow \exists \delta \in(0,1) \text { s.t. } x, y \in S_{X},\|x-y\| / 2>1-\delta \Rightarrow\|x+y\| / 2 \leq 1-\delta \\
& \Leftrightarrow \exists \delta \in(0,1) \text { s.t. } x, y \in S_{X},\|x-y\|>2-2 \delta \Rightarrow 1-\|x+y\| / 2 \geq \delta \\
& \Leftrightarrow \exists \delta \in(0,1) \text { s.t. } \delta_{X}(2-2 \delta) \geq \delta .
\end{aligned}
$$

(i) $\Rightarrow$ (ii). If $X$ is uniformly non-square, then by taking $\varepsilon=2-2 \delta \in(0,2)$ in the above equivalences, we obtain $\delta_{X}(\varepsilon) \geq 1-\varepsilon / 2>0$.

(ii) $\Rightarrow$ (i). Assume that there exists $\varepsilon_{0} \in(0,2)$ such that $\delta_{X}\left(\varepsilon_{0}\right)>0$, i.e. $\delta_{X}\left(\varepsilon_{0}\right) \geq \eta_{0}>0$ for some $\eta_{0} \in(0,1)$. If we set $2-2 \delta=\varepsilon \in\left[\varepsilon_{0}, 2\right)$, then $\delta \in\left(0,1-\varepsilon_{0} / 2\right]$ and

$$
\delta_{X}(2-2 \delta)=\delta_{X}(\varepsilon) \geq \delta_{X}\left(\varepsilon_{0}\right) \geq \eta_{0}>0,
$$

which means that, for any $x, y \in S_{X}$, if $\|x-y\| \geq 2-2 \delta$, then $1-\|x+y\| / 2 \geq$ $\eta_{0}$. Let $\delta^{\prime}=\min \left\{\delta, \eta_{0}\right\}$. Of course, $\delta^{\prime} \in(0,1)$. We want to show that either

$$
\|x-y\| / 2 \leq 1-\delta^{\prime} \quad \text { or } \quad\|x+y\| / 2 \leq 1-\delta^{\prime} .
$$

If $\|x-y\| / 2 \leq 1-\delta^{\prime}$, then we are done. Assume then that $\|x-y\| / 2>1-\delta$. This gives $\|x-y\|>2\left(1-\delta^{\prime}\right) \geq 2(1-\delta)$ and by the above assumption we get $1-\|x+y\| / 2 \geq \eta_{0}$ or $\|x+y\| / 2 \leq 1-\eta_{0} \leq 1-\delta^{\prime}$. This shows that $X$ is uniformly non-square.

(ii) $\Leftrightarrow$ (iii) follows from the definition of $\varepsilon_{0}(X)$, and (i) $\Leftrightarrow($ iv) is again only the definition.

$(\mathrm{v}) \Leftrightarrow(\mathrm{vi})$ is a consequence of Lemma 1 below, and (v) $\Leftrightarrow$ (vii) follows from the fact that $\varrho_{X}(\tau) / \tau$ is increasing.

(vii) $\Leftrightarrow$ (viii) and (ix) $\Leftrightarrow($ iii) follow directly from the formulas

$$
\varepsilon_{0}\left(X^{*}\right)=2 \varrho_{X}(0)=2 \lim _{\tau \rightarrow 0} \frac{\varrho_{X}(\tau)}{\tau}, \quad \varepsilon_{0}(X)=2 \varrho_{X^{*}}^{\prime}(0)=2 \lim _{\tau \rightarrow 0} \frac{\varrho_{X^{*}}(\tau)}{\tau},
$$

which are consequences of (1.4), (1.5), and this was observed by Turett [40] and Khamsi [27] (cf. also [1], Corollary 3.12).

Lemma 1. The modulus of smoothness of any Banach space $X$ satisfies either the equality $\varrho_{X}(\tau)=\tau$ for all $\tau>0$ or the strict inequality $\varrho_{X}(\tau)<\tau$ for all $\tau>0$.

Proof. It is enough to show that if $\varrho_{X}\left(\tau_{0}\right)=\tau_{0}$ for a certain $\tau_{0}>0$, then $\varrho_{X}(\tau)=\tau$ for all $\tau>0$. Since $\varrho_{X}(\tau) / \tau$ is increasing and $\varrho_{X}(\tau) \leq \tau$, it follows that for $\tau \geq \tau_{0}$ we have $1=\varrho_{X}\left(\tau_{0}\right) / \tau_{0} \leq \varrho_{X}(\tau) / \tau \leq 1$, i.e., 
$\varrho_{X}(\tau)=\tau$ for $\tau \geq \tau_{0}$. Let $0<\tau<\tau_{0}$ and assume that $\varrho_{X}(\tau)<\tau$. Then, by the convexity of $\varrho_{X}$, we have, for $\tau_{1}>\tau_{0}$,

$$
\begin{aligned}
\tau_{0}=\varrho_{X}\left(\tau_{0}\right) & =\varrho_{X}\left(\frac{\tau_{0}-\tau}{\tau_{1}-\tau} \tau_{1}+\frac{\tau_{1}-\tau_{0}}{\tau_{1}-\tau} \tau\right) \\
& \leq \frac{\tau_{0}-\tau}{\tau_{1}-\tau} \varrho_{X}\left(\tau_{1}\right)+\frac{\tau_{1}-\tau_{0}}{\tau_{1}-\tau} \varrho_{X}(\tau) \\
& <\frac{\tau_{0}-\tau}{\tau_{1}-\tau} \tau_{1}+\frac{\tau_{1}-\tau_{0}}{\tau_{1}-\tau} \tau=\tau_{0},
\end{aligned}
$$

a contradiction. Therefore $\varrho_{X}(\tau)=\tau$.

REMARK 2. A uniformly non-square Banach space is super-reflexive (James [20], cf. also [3]), i.e., there exists an equivalent norm $\|\cdot\|$ on $X$ in which $(X,\|\cdot\|)$ is uniformly convex. The converse implication is not true in the sense that there exists an equivalent norm $|\cdot|$ on $X$ for which $\delta_{(X,|\cdot|)}(\varepsilon)=0$ for all $0<\varepsilon<2$.

Example 1. Let $1<p<\infty$ and consider the new norms in $\ell_{p}$ :

$$
\begin{gathered}
\|x\|=\max \left\{\left|x_{1}\right|+\left|x_{2}\right|,\left(\sum_{k=3}^{\infty}\left|x_{k}\right|^{p}\right)^{1 / p}\right\}, \\
\|x\|^{\prime}=\max \left\{\left|x_{1}\right|,\left|x_{2}\right|,\left(\sum_{k=3}^{\infty}\left|x_{k}\right|^{p}\right)^{1 / p}\right\} .
\end{gathered}
$$

Then $\|x\|_{p} \leq 2^{1 / p}\|x\| \leq 2^{1 / p+1}\|x\|^{\prime} \leq 2^{1 / p+1}\|x\| \leq 4\|x\|_{p}$, and since $\ell_{p}$ is uniformly convex it follows that the spaces $X=\left(\ell_{p},\|\cdot\|\right)$ and $X=\left(\ell_{p},\|\cdot\|^{\prime}\right)$ are super-reflexive but not uniformly non-square since $\delta_{X}(\varepsilon)=0$ for all $0<\varepsilon<2$. To show the last equality it is enough to take $x=(1,0,0, \ldots)$ and $y=(0,1,0, \ldots)$. This example shows that there exists a super-reflexive Banach space such that $\delta_{X}(\varepsilon)=0$ for all $0<\varepsilon<2$. More generally, if $X=(X,\|\cdot\|)$ is a non-trivial Banach space and $X_{1}$ is a two-dimensional subspace of $X$, then $X=X_{1} \oplus X_{2}$ and on $X_{1}$ we can take any equivalent norm $\|x\|_{1}$ (for example, $\|x\|_{1}=\left|x_{1}\right|+\left|x_{2}\right|$ ) such that $\delta_{\left(X,\|\cdot\|_{1}\right)}(\varepsilon)=0$ for all $0<\varepsilon<2$. Let $\|\cdot\|_{2}$ be the norm $\|\cdot\|$ restricted to $X_{2}$. Then, clearly, $\|x\|=\max \left\{\|x\|_{1},\|x\|_{2}\right\}$ is equivalent to $\|\cdot\|$ and $\delta_{(X,\|\cdot\|)}(\varepsilon)=0$ for all $0<\varepsilon<2$. Therefore we can formulate a corollary:

COROllary 1. For any non-trivial Banach space $X$ there is an equivalent norm $\|\cdot\|$ on $X$ such that $(X,\|\cdot\|)$ is not uniformly non-square.

Let us collect properties of the James constant $J(X)$ for non-trivial Banach spaces (see Casini [6], Gao-Lau [15] and [16]):

(i) $\sqrt{2} \leq J(X) \leq 2$.

(ii) $J(X)=\sqrt{2}$ if $X$ is a Hilbert space; the converse is not true. 
(iii) If $1 \leq p \leq \infty$ and $\operatorname{dim} L_{p}(\mu) \geq 2$, then $J\left(L_{p}(\mu)\right)=\max \left\{2^{1 / p}, 2^{1-1 / p}\right\}$. Casini [6, Prop. 2.2] and also Gao-Lau [15, Th. 3.3] and [16, Th. 5.4] proved that

$$
J(X)=\sup \left\{\varepsilon \in(0,2): \delta_{X}(\varepsilon) \leq 1-\varepsilon / 2\right\} .
$$

(iv) $J(X)=\sup \left\{\min (\|x+y\|,\|x-y\|): x, y \in B_{X}\right\}$.

REMARK 3. In the one-dimensional real case, i.e., when $X=(\mathbb{R},|\cdot|)$, we have $J(X)=0$ and $J_{1}(X)=\sup \left\{\min (\|x+y\|,\|x-y\|): x, y \in B_{X}\right\}=1$.

We give an example of a two-dimensional Banach space $X$ for which $J\left(X^{*}\right) \neq J(X)$.

Example 2. Let $X=\mathbb{R}^{2}$ with the $\ell_{2}-\ell_{1}$ norm defined by

$$
\|x\|= \begin{cases}\|x\|_{2} & \text { if } x_{1} x_{2} \geq 0 \\ \|x\|_{1} & \text { if } x_{1} x_{2} \leq 0\end{cases}
$$

where $\|x\|_{p}=\left(\left|x_{1}\right|^{p}+\left|x_{2}\right|^{p}\right)^{1 / p}$ for $p=1,2$. Then $\delta_{X}(\varepsilon)=0$ if $0 \leq \varepsilon \leq$ $\sqrt{2}, \delta_{X}(\varepsilon)=1-\sqrt{2-\varepsilon^{2} / 2}$ if $\sqrt{2} \leq \varepsilon \leq \sqrt{8 / 3}$, and $\delta_{X}(\varepsilon)=1-\sqrt{1-\varepsilon^{2} / 8}$ if $\sqrt{8 / 3} \leq \varepsilon \leq 2$ (see Goebel-Kirk [17], Ex. 5.8). Therefore, by (2.2), $J(X)=$ $\sqrt{8 / 3}$. The dual space $X^{*}$ of $X$ has the $\ell_{2}-\ell_{\infty}$ norm

$$
\|x\|^{*}= \begin{cases}\|x\|_{2} & \text { if } x_{1} x_{2} \geq 0 \\ \|x\|_{\infty} & \text { if } x_{1} x_{2} \leq 0\end{cases}
$$

For the vectors $x=(-1,1)$ and $y=(1 / \sqrt{2}, 1 / \sqrt{2})$ from the unit sphere $S_{X^{*}}$ we have $\|x+y\|^{*}=\|x-y\|^{*}=1+1 / \sqrt{2}$, and so

$$
J\left(X^{*}\right) \geq 1+1 / \sqrt{2}>\sqrt{8 / 3}=J(X) .
$$

We now show the relation between the James constants for a space and for its dual, which answers a question of Gao and Lau [16], Question 7.3.

Theorem 1. For any Banach space $X$ we have

$$
2 J(X)-2 \leq J\left(X^{*}\right) \leq J(X) / 2+1 .
$$

Proof. First observe that if $a, b \in S_{X}$, then

$$
\|a+b\|+\|a-b\| \leq \min \{\|a+b\|,\|a-b\|\}+2 .
$$

From the definition of $J(X)$ for any $\varepsilon>0$ we can find $x, y \in S_{X}$ such that

$$
\min \{\|x+y\|,\|x-y\|\} \geq J(X)-\varepsilon .
$$

Moreover, by using the Hahn-Banach theorem we can find $u^{*}, v^{*} \in S_{X^{*}}$ such that

$$
u^{*}(x+y)=\|x+y\| \quad \text { and } \quad v^{*}(x-y)=\|x-y\| .
$$


Then

$$
\begin{aligned}
J\left(X^{*}\right) & \geq \min \left\{\left\|u^{*}+v^{*}\right\|,\left\|u^{*}-v^{*}\right\|\right\} \\
& \geq\left\|u^{*}+v^{*}\right\|+\left\|u^{*}-v^{*}\right\|-2 \geq\left(u^{*}+v^{*}\right)(x)+\left(u^{*}-v^{*}\right)(y)-2 \\
& =u^{*}(x+y)+v^{*}(x-y)-2=\|x+y\|+\|x-y\|-2 \\
& \geq 2 \min \{\|x+y\|,\|x-y\|\}-2 \geq 2\{J(X)-\varepsilon\}-2 .
\end{aligned}
$$

Since $\varepsilon>0$ was arbitrary we obtain

$$
J\left(X^{*}\right) \geq 2 J(X)-2,
$$

and the first inequality of (2.3) is proved. To obtain the other inequality, let $u^{*}, v^{*} \in S_{X^{*}}$ and suppose $\varepsilon>0$. Then there exist $x, y \in S_{X}$ such that

$$
\left(u^{*}+v^{*}\right)(x)>\left\|u^{*}+v^{*}\right\|-\varepsilon \text { and }\left(u^{*}-v^{*}\right)(y)>\left\|u^{*}-v^{*}\right\|-\varepsilon .
$$

Thus

$$
\begin{aligned}
\min & \left\{\left\|u^{*}+v^{*}\right\|,\left\|u^{*}-v^{*}\right\|\right\} \leq\left(\left\|u^{*}+v^{*}\right\|+\left\|u^{*}-v^{*}\right\|\right) / 2 \\
& <\left[\left(u^{*}+v^{*}\right)(x)+\left(u^{*}-v^{*}\right)(y)+2 \varepsilon\right] / 2=\left[u^{*}(x+y)+v^{*}(x-y)+2 \varepsilon\right] / 2 \\
& \leq\left[\left\|u^{*}\right\| \cdot\|x+y\|+\left\|v^{*}\right\| \cdot\|x-y\|+2 \varepsilon\right] / 2=[\|x+y\|+\|x-y\|+2 \varepsilon] / 2 \\
& \leq[\min \{\|x+y\|,\|x-y\|\}+2+2 \varepsilon] / 2 \leq[J(X)+2+2 \varepsilon] / 2 .
\end{aligned}
$$

From this the second inequality readily follows.

REMARK 4. If $X$ is not uniformly non-square (for example $X=\ell_{1}, \ell_{\infty}$ or $\left.c_{0}\right)$, then $J(X)=2$ and we have equalities in (2.3):

$$
2 J(X)-2=J\left(X^{*}\right)=J(X) / 2+1=2 .
$$

As an immediate consequence of Proposition 1 and Theorem 1 we have a result proved by several authors (implicitly James [21] and explicitly e.g. Fitzpatrick-Reznick [14] and Takahashi-Kato [38]; cf. also our Proposition 1, the equivalence of (i) with (iii) and (viii)).

COROllary 2. A Banach space $X$ is uniformly non-square if and only if its dual $X^{*}$ is uniformly non-square.

Let us mention that Schäffer (1970) introduced another definition of uniform non-squareness. $X$ is called uniformly non-square (in the sense of Schäffer) if there exists $\lambda>1$ such that for every $x, y \in S_{X}$ we have either $\|x+y\|>\lambda$ or $\|x-y\|>\lambda$. The constant

$$
S(X)=\inf \left\{\max (\|x+y\|,\|x-y\|): x, y \in S_{X}\right\}
$$

is called the Schäffer constant of the space $X$. James's and Schäffer's definitions are equivalent in the sense that $J(X)<2$ if and only if $S(X)>1$. This follows from the equality below (the method of proof is taken from Casini [6], Proposition 2.1):

Theorem 2. If $X$ is a non-trivial Banach space, then $J(X) S(X)=2$. 
Proof. For any $x, y \in S_{X}, x \neq \pm y$, let

Then $u, v \in S_{X}$, and

$$
u=\frac{x+y}{\|x+y\|}, \quad v=\frac{x-y}{\|x-y\|} .
$$

$$
u \pm v=\left(\frac{1}{\|x+y\|} \pm \frac{1}{\|x-y\|}\right) x+\left(\frac{1}{\|x+y\|} \mp \frac{1}{\|x-y\|}\right) y
$$

Therefore

$$
\begin{aligned}
\|u \pm v\| & \geq|\|x\|| \frac{1}{\|x+y\|} \pm \frac{1}{\|x-y\|}|-\|y\|| \frac{1}{\|x+y\|} \mp \frac{1}{\|x-y\|} \| \\
& =\left\|\frac{1}{\|x+y\|} \pm \frac{1}{\|x-y\|}|-| \frac{1}{\|x+y\|} \mp \frac{1}{\|x-y\|}\right\| \\
& =2 \min \left(\frac{1}{\|x+y\|}, \frac{1}{\|x-y\|}\right)=\frac{2}{\max (\|x+y\|,\|x-y\|)}
\end{aligned}
$$

and so

$$
J(X) \geq \min (\|u+v\|,\|u-v\|) \geq \frac{2}{\max (\|x+y\|,\|x-y\|)}
$$

for all $x, y \in S_{X}, x \neq \pm y$, i.e., $J(X) \geq 2 / S(X)$. Also

$$
\begin{aligned}
\|u \pm v\| & \leq\|x\|\left|\frac{1}{\|x+y\|} \pm \frac{1}{\|x-y\|}\right|+\|y\|\left|\frac{1}{\|x+y\|} \mp \frac{1}{\|x-y\|}\right| \\
& =\left|\frac{1}{\|x+y\|} \pm \frac{1}{\|x-y\|}\right|+\left|\frac{1}{\|x+y\|} \mp \frac{1}{\|x-y\|}\right| \\
& =2 \max \left(\frac{1}{\|x+y\|}, \frac{1}{\|x-y\|}\right)=\frac{2}{\min (\|x+y\|,\|x-y\|)},
\end{aligned}
$$

and so

$$
S(X) \leq \max (\|u+v\|,\|u-v\|) \leq \frac{2}{\min (\|x+y\|,\|x-y\|)}
$$

for all $x, y \in S_{X}, x \neq \pm y$, i.e., $S(X) \leq 2 / J(X)$. Combining the above inequalities, we obtain $J(X) S(X)=2$.

REMARK 5. If $X=(\mathbb{R},|\cdot|)$, then $J(X)=0$ and $S(X)=2$.

3. Jordan-von Neumann constant and James constant. The Jordan-von Neumann constant $C_{\mathrm{NJ}}(X)$ of a Banach space $X$ is defined by

$$
C_{\mathrm{NJ}}(X)=\sup \left\{\frac{\|x+y\|^{2}+\|x-y\|^{2}}{2\left(\|x\|^{2}+\|y\|^{2}\right)}: x, y \in X, \text { not both zero }\right\} .
$$

Since usually this constant is called the von Neumann-Jordan constant we will keep the notation $C_{\mathrm{NJ}}(X)$. Let us collect its properties (see Jordan-von Neumann [23], Clarkson [8], Kato-Takahashi [25], [26] and [38]): 
(i) $1 \leq C_{\mathrm{NJ}}(X) \leq 2$.

(ii) $X$ is a Hilbert space if and only if $C_{\mathrm{NJ}}(X)=1$.

(iii) $X$ is uniformly non-square if and only if $C_{\mathrm{NJ}}(X)<2$.

(iv) $C_{\mathrm{NJ}}(X)=C_{\mathrm{NJ}}\left(X^{*}\right)$.

(v) If $1 \leq p \leq \infty$ and $\operatorname{dim} L_{p}(\mu) \geq 2$, then $C_{\mathrm{NJ}}\left(L_{p}(\mu)\right)=2^{2 / r-1}$ with $r=\min \left\{p, p^{\prime}\right\}$.

Theorem 3. For any non-trivial Banach space $X$,

$$
\frac{1}{2} J(X)^{2} \leq C_{\mathrm{NJ}}(X) \leq \frac{J(X)^{2}}{(J(X)-1)^{2}+1} .
$$

Proof. For any $x, y \in S_{X}$, we have

$$
\begin{aligned}
\min \left\{\|x+y\|^{2},\|x-y\|^{2}\right\} & \leq \frac{\|x+y\|^{2}+\|x-y\|^{2}}{2} \\
& =2 \frac{\|x+y\|^{2}+\|x-y\|^{2}}{2\left(\|x\|^{2}+\|y\|^{2}\right)} \leq 2 C_{\mathrm{NJ}}(X),
\end{aligned}
$$

which implies the left inequality of (3.1). To prove the right inequality we first observe that

$$
C_{\mathrm{NJ}}(X)=\sup \left\{\frac{\|x+y\|^{2}+\|x-y\|^{2}}{2\left(\|x\|^{2}+\|y\|^{2}\right)}: x, y \in X,\|x\|=1,\|y\| \leq 1\right\} .
$$

In fact, if $0 \neq\|x\| \geq\|y\|$ (for $\|x\| \leq\|y\| \neq 0$ the proof is similar), then

$$
\|x \pm y\|=\|x\|\left\|\frac{x}{\|x\|} \pm \frac{y}{\|x\|}\right\|
$$

and so

$$
\frac{\|x+y\|^{2}+\|x-y\|^{2}}{2\left(\|x\|^{2}+\|y\|^{2}\right)}=\frac{\left\|\frac{x}{\|x\|}+\frac{y}{\|x\|}\right\|^{2}+\left\|\frac{x}{\|x\|}-\frac{y}{\|x\|}\right\|^{2}}{2\left(1+\left\|\frac{y}{\|x\|}\right\|^{2}\right)},
$$

which shows that the supremum in the definition of $C_{\mathrm{NJ}}(X)$ can be taken just over $x, y \in X$ such that $\|x\|=1$ and $\|y\| \leq 1$.

To obtain the right estimate in (3.1) we consider two cases (of course for $\|x\|=1$ and $\|y\| \leq 1)$ :

CASE 1: $\|y\|=t \geq J(X)-1$. Then

$$
\begin{aligned}
A:=\frac{\|x+y\|^{2}+\|x-y\|^{2}}{2\left(\|x\|^{2}+\|y\|^{2}\right)} & \leq \frac{(\|x\|+\|y\|)^{2}+(\min \{\|x+y\|,\|x-y\|\})^{2}}{2\left(\|x\|^{2}+\|y\|^{2}\right)} \\
& \leq \frac{(1+t)^{2}+J(X)^{2}}{2\left(1+t^{2}\right)}=: f(t) .
\end{aligned}
$$


Since $f^{\prime}(t)=\left(1-t J(X)^{2}-t^{2}\right) /\left(1+t^{2}\right)^{2}$ it follows that $f$ is increasing on $\left(0, t_{0}\right)$ and decreasing on $\left(t_{0}, 1\right)$, where $t_{0}=\left(-J(X)^{2}+\sqrt{J(X)^{4}+4}\right) / 2$. Now $J(X)-1 \geq t_{0}$ and so

$$
A \leq f(t) \leq f(J(X)-1)=\frac{J(X)^{2}}{(J(X)-1)^{2}+1} .
$$

Case $2:\|y\|=t \leq J(X)-1$. Then

$$
A \leq \frac{2(\|x\|+\|y\|)^{2}}{2\left(\|x\|^{2}+\|y\|^{2}\right)}=\frac{(1+t)^{2}}{1+t^{2}}=: g(t) .
$$

Since $g^{\prime}(t)=2\left(1-t^{2}\right) /\left(1+t^{2}\right)^{2}$ it follows that $g$ is increasing on $(0,1]$ and so

$$
A \leq g(t) \leq g(J(X)-1)=\frac{J(X)^{2}}{(J(X)-1)^{2}+1} .
$$

Thus, in both cases,

$$
A \leq \frac{J(X)^{2}}{(J(X)-1)^{2}+1} .
$$

This completes the proof.

REMARK 6. (a) Immediately from Proposition 1 and Theorem 3 we obtain the result of (iii) above: a non-trivial Banach space $X$ is uniformly non-square if and only if $C_{\mathrm{NJ}}(X)<2$.

(b) Concerning equalities and strict inequalities in (3.1), we have:

(i) If $X$ is not uniformly non-square, then $J(X)=C_{\mathrm{NJ}}(X)=2$ and we have both equalities in (3.1): $J(X)^{2} / 2=C_{\mathrm{NJ}}(X)=J(X)^{2} /\left[(J(X)-1)^{2}+1\right]$ $=2$.

(ii) For a Hilbert space $H$, we have $J(H)^{2} / 2=C_{\mathrm{NJ}}(H)=1$ and so $J(H)^{2} /\left[(J(H)-1)^{2}+1\right] \approx 1.7$.

(iii) If $1 \leq p \leq \infty$ and $\operatorname{dim} L_{p}(\mu) \geq 2$, then $J\left(L_{p}(\mu)\right)=2^{1 / r}$ and $C_{\mathrm{NJ}}\left(L_{p}(\mu)\right)=2^{2 / r-1}$, with $r=\min \left\{p, p^{\prime}\right\}$, from which we obtain $J\left(L_{p}(\mu)\right)^{2} / 2$ $=C_{\mathrm{NJ}}\left(L_{p}(\mu)\right)$. Also for the space $X=L_{p}\left[L_{q}\right]$ (the $L_{q}$-valued $L_{p}$-space) we have the same equalities with $r=\min \left\{p, p^{\prime}, q, q^{\prime}\right\}$.

(iv) There are Lorentz sequence spaces $X=\ell_{p, 2}$ for which we have the equality $J(X)^{2} / 2=C_{\mathrm{NJ}}(X)$.

ExAmple 3 . Let $\ell_{p, 2}(2 \leq p<\infty)$ be a two-dimensional Lorentz sequence space with the norm

$$
\|x\|_{p, 2}=\left(x_{1}^{* 2}+2^{2 / p-1} x_{2}^{* 2}\right)^{1 / 2},
$$

where $\left(x_{1}^{*}, x_{2}^{*}\right)$ is the rearrangement of $\left(\left|x_{1}\right|,\left|x_{2}\right|\right)$ such that $x_{1}^{*} \geq x_{2}^{*}$ (cf. [29]). We have

$$
J\left(\ell_{p, 2}\right)=\frac{2}{\left(1+2^{2 / p-1}\right)^{1 / 2}} \quad \text { and } \quad C_{\mathrm{NJ}}\left(\ell_{p, 2}\right)=\frac{2}{1+2^{2 / p-1}} .
$$


Let us show (3.3). First, observe that $\|x\|_{2} \leq\left[2 /\left(1+2^{2 / p-1}\right)\right]^{1 / 2}\|x\|_{p, 2}$. Then

$$
\begin{aligned}
\|x+y\|_{p, 2}^{2}+\|x-y\|_{p, 2}^{2} & \leq\left(\|x+y\|_{2}^{2}+\|x-y\|_{2}^{2}\right)=2\left(\|x\|_{2}^{2}+\|y\|_{2}^{2}\right) \\
& \leq \frac{2}{1+2^{2 / p-1}} 2\left(\|x\|_{p, 2}^{2}+\|y\|_{p, 2}^{2}\right)
\end{aligned}
$$

or

$$
C_{\mathrm{NJ}}\left(\ell_{p, 2}\right) \leq 2 /\left(1+2^{2 / p-1}\right) .
$$

On the other hand, for $x_{0}=(\alpha, \alpha)$ and $y_{0}=(\alpha,-\alpha)$ with $\alpha=1 /(1+$ $\left.2^{2 / p-1}\right)^{1 / 2}$, we have

$$
C_{\mathrm{NJ}}\left(\ell_{p, 2}\right) \geq \frac{\left\|x_{0}+y_{0}\right\|_{p, 2}^{2}+\left\|x_{0}-y_{0}\right\|_{p, 2}^{2}}{2\left(\left\|x_{0}\right\|_{p, 2}^{2}+\left\|y_{0}\right\|_{p, 2}^{2}\right)}=2 \alpha^{2}=\frac{2}{1+2^{2 / p-1}} .
$$

Also $J\left(\ell_{p, 2}\right) \geq 2 \alpha=2 /\left(1+2^{2 / p-1}\right)^{1 / 2}$, and by the first estimate in (3.1) we obtain

$$
J(X) \leq \sqrt{2 C_{\mathrm{NJ}}(X)}=2 \alpha=2 /\left(1+2^{2 / p-1}\right)^{1 / 2},
$$

and the proof of (3.3) is complete.

REmark 7. There exists a two-dimensional Banach space $X$ for which $J(X)^{2} / 2<C_{\mathrm{NJ}}(X)$.

EXAmple $4\left(\ell_{\infty}-\ell_{1}\right.$ norm). Let $X=\mathbb{R}^{2}$ with the norm defined by

$$
\|x\|= \begin{cases}\|x\|_{\infty} & \text { if } x_{1} x_{2} \geq 0 \\ \|x\|_{1} & \text { if } x_{1} x_{2} \leq 0\end{cases}
$$

Then $\delta_{X}(\varepsilon)=\max \{0,(\varepsilon-1) / 2\}$ and $\varrho_{X}(\tau)=\max \{\tau / 2, \tau-1 / 2\}$. Therefore, by $(2.2)$,

$$
J(X)=3 / 2 \text {. }
$$

If we take $x=(1,1)$ and $y=(0,1)$, then $C_{\mathrm{NJ}}(X) \geq 5 / 4$ and so

$$
J(X)^{2} / 2=9 / 8<5 / 4 \leq C_{\mathrm{NJ}}(X) .
$$

Note that the dual space $X^{*}$ of $X$ is given by the $\ell_{1}-\ell_{\infty}$ norm and is isometric to $X$.

Remark 8. If $\operatorname{dim} X=2$, then $C_{\mathrm{NJ}}(X)=2$ if and only if $X$ is isometric to $\ell_{\infty}^{2}$. If $\operatorname{dim} X<\infty$, then $C_{\mathrm{NJ}}(X)=2$ if and only if $X$ contains a twodimensional subspace isometric to $\ell_{\infty}^{2}$.

\section{Jordan-von Neumann constant and the normal structure} coefficient. A Banach space $X$ is said to have normal structure if $r(K)<$ $\operatorname{diam}(K)$ for every non-singleton closed bounded convex subset $K$ of $X$, where $\operatorname{diam}(K):=\sup \{\|x-y\|: x, y \in K\}$ is the diameter of $K$ and $r(K):=\inf \{\sup \{\|x-y\|: y \in K\}: x \in K\}$ is the Chebyshev radius of $K$. The normal structure coefficient (Bynum, 1980) of $X$ is the number $N(X)=\inf \{\operatorname{diam}(K) / r(K): K \subset X$ bounded and convex, $\operatorname{diam}(K)>0\}$. 
Obviously, $1 \leq N(X) \leq 2$. The space $X$ is said to have uniform normal structure if $N(X)>1$. A Banach space $X$ is said to have the fixed point property (FPP) (for non-expansive mappings) provided for any non-empty bounded convex subset $K$ of $X$, every non-expansive mapping $T: K \rightarrow K$ has a fixed point. The following results are well known (see Goebel-Kirk [17], Th. 4.1 and 4.4):

(a) If $X$ is a reflexive space and has normal structure, then $X$ has FPP (Kirk, 1965).

(b) If $X$ has uniform normal structure, then $X$ is reflexive and hence $X$ has FPP.

We now collect some properties of the normal structure coefficient (see Bynum [5], Maluta [32], Domínguez Benavides [11]; cf. also [17], [1] and [18]):

(i) $N(X) \geq 1 /\left(1-\delta_{X}(1)\right)$; in particular, if $X$ is uniformly convex, then $N(X)>1$ and $X$ has FPP.

(ii) If $X$ is non-reflexive, then $N(X)=1$.

(iii) If $\operatorname{dim} X=\infty$, then $N(X) \leq \sqrt{2}$.

(iv) If $1 \leq p \leq \infty$ and a $\sigma$-finite measure space $(\Omega, \Sigma, \mu)$ is such that $\operatorname{dim} L_{p}(\mu)=\infty$, then $N\left(L_{p}(\mu)\right)=\min \left\{2^{1 / p}, 2^{1-1 / p}\right\}$.

Gao and Lau [16] showed that if $J(X)<3 / 2$ (or equivalently $\delta_{X}(3 / 2)>$ $1 / 4$ ), then $X$ has uniform normal structure. Prus [36] gave an estimate of $N(X)$ by $J(X)$ which contains Gao-Lau's and Bynum's results: For any non-trivial Banach space $X$,

$$
N(X) \geq J(X)+1-\left\{(J(X)+1)^{2}-4\right\}^{1 / 2} .
$$

We present here the following estimate of $N(X)$ by the $C_{\mathrm{NJ}}(X)$-constant:

TheOREM 4. For any non-trivial Banach space $X$,

$$
N(X) \geq\left(C_{\mathrm{NJ}}(X)-1 / 4\right)^{-1 / 2} .
$$

Proof. Let $K$ be an arbitrary bounded convex subset of $X$ with $d:=$ $\operatorname{diam}(K)>0$. Then for any $\varepsilon>0$ there exist $u$ and $v$ in $K$ such that

$$
\|u-v\|>d(1-\varepsilon) \text {. }
$$

Put $z=(u+v) / 2 \in K$. For any $x \in K$ consider $\xi=x-u$ and $\eta=x-v$. Then since

$$
\frac{\|\xi+\eta\|^{2}+\|\xi-\eta\|^{2}}{2\left(\|\xi\|^{2}+\|\eta\|^{2}\right)} \leq C_{\mathrm{NJ}}(X)
$$

it follows that

$$
\frac{\|2 x-u-v\|^{2}+\|-u+v\|^{2}}{2\left(\|x-u\|^{2}+\|x-v\|^{2}\right)} \leq C_{\mathrm{NJ}}(X)
$$


and hence,

$$
\begin{aligned}
\|x-z\|^{2} & \leq \frac{1}{2} C_{\mathrm{NJ}}(X)\left(\|x-u\|^{2}+\|x-v\|^{2}\right)-\frac{1}{4}\|-u+v\|^{2} \\
& \leq d^{2} C_{\mathrm{NJ}}(X)-\frac{1}{4} d^{2}(1-\varepsilon)^{2}
\end{aligned}
$$

Thus

$$
r(K) \leq \sup \{\|x-z\|: x \in K\} \leq d\left\{C_{\mathrm{NJ}}(X)-\frac{1}{4}(1-\varepsilon)^{2}\right\}^{1 / 2} .
$$

Letting $\varepsilon \rightarrow 0$, we obtain (4.2).

Theorem 4 and the fact that $C_{\mathrm{NJ}}(X)=C_{\mathrm{NJ}}\left(X^{*}\right)$ yield in a straightforward way the corollary below. We should also mention here that the normal and uniform normal structures are not inherited by the dual spaces in general (cf. [4], [37]).

Corollary 3. If $C_{\mathrm{NJ}}(X)<5 / 4$, then $X$ as well as its dual $X^{*}$ have uniform normal structure, and hence $X$ and $X^{*}$ have the fixed point property.

REMARK 9. The assumption $C_{\mathrm{NJ}}(X)<5 / 4$ implies, by the first inequality in (3.1), that $J(X) \leq \sqrt{2 C_{\mathrm{NJ}}(X)}<\sqrt{10} / 2$ and this is a little weaker than Gao-Lau's condition $J(X)<3 / 2$. Gao and Lau asked in [16], Question 7.1, whether the estimate $J(X)<3 / 2$ is sharp for $X$ to have uniform normal structure. Note that in the class of $X$ for which $J(X)^{2} / 2=C_{\mathrm{NJ}}(X)$ the constant $3 / 2$ is not sharp since, by Theorem $4, J(X)<\sqrt{10} / 2$ implies that $X$ has uniform normal structure.

REMARK 10. Our estimate (4.2), like any other known estimates, is still not very sharp in certain Banach spaces. For instance, $N\left(\ell_{2}\right)=\sqrt{2}$ and the estimate $(4.2)$ gives $N\left(\ell_{2}\right) \geq(1-1 / 4)^{-1 / 2}=2 / \sqrt{3}$. On the other hand, the bound (4.2) gives information about the uniform normal structure of $X$ when $C_{\mathrm{NJ}}(X)<5 / 4$ but not when $C_{\mathrm{NJ}}(X)$ is close to 2 .

The following example shows that there exists a Banach space with uniform normal structure whose $C_{\mathrm{NJ}}$-constant is arbitrarily near to 2 . This space is even not strictly convex and it is also an example of a uniformly convex (Hilbert) space which has an equivalent norm without normal structure.

ExAmple 5 . For $\lambda \geq 1$ let $X_{\lambda}$ be the space $\ell_{2}$ (cf. [2], Th. 2; [3], p. 213, and [1], pp. 145-146) with the norm

$$
|x|_{\lambda}=\max \left\{\|x\|_{2}, \lambda\|x\|_{\infty}\right\} .
$$

Then, since $\|x\|_{2} \leq|x|_{\lambda} \leq \lambda\|x\|_{2}$ it follows that $X_{\lambda}$ is reflexive. Moreover,

$$
C_{\mathrm{NJ}}\left(X_{\lambda}\right)=\min \left\{2, \lambda^{2}\right\}, \quad J\left(X_{\lambda}\right)=\min \{2, \lambda \sqrt{2}\}
$$


(cf. Kato-Takahashi [25], Proposition 1) and by [5] together with the considerations in [1], p. 146,

$$
N\left(X_{\lambda}\right)=\max \{1, \sqrt{2} / \lambda\}
$$

Thus $X_{\lambda}$ has uniform normal structure if and only if $\lambda<\sqrt{2}$. The space $X_{\lambda}$ has normal structure if and only if $\lambda<\sqrt{2}$ (cf. [2], Th. 2), and it has FPP for all $\lambda \geq 1$ (cf. Goebel-Kirk [17], Ex. 6.3, Bynum [5], Th. 6, and [18], p. 772). We mention here that one of the first who considered the space $X_{\sqrt{2}}$ as an example of a reflexive Banach space with FPP but without normal structure was Karlovitz in 1976 (cf. [2] and [18]). Note that $X_{\lambda}$ is not strictly convex for $\lambda>1$ and $C_{\mathrm{NJ}}\left(X_{\lambda}\right)=\lambda^{2} \rightarrow 2$ as $\lambda \rightarrow \sqrt{2}$. Moreover, $X_{\lambda}$ is the Orlicz sequence space $\ell_{\varphi_{\lambda}}$ generated by the convex function $\varphi_{\lambda}(u)=u^{2}$ for $0 \leq u \leq 1 / \lambda$ and $\varphi_{\lambda}(u)=\infty$ for $u>1 / \lambda$ (cf. [31]).

\section{Banach-Mazur distance and stability under norm perturba-} tions. For isomorphic Banach spaces $X$ and $Y$, the Banach-Mazur distance between $X$ and $Y$, denoted by $d(X, Y)$, is defined to be the infimum of $\|T\| \cdot\left\|T^{-1}\right\|$ taken over all bicontinuous linear operators $T$ from $X$ onto $Y$ (cf. [39]). Bynum [5] proved that if $X$ and $Y$ are isomorphic Banach spaces, then

$$
\frac{N(X)}{d(X, Y)} \leq N(Y) \leq N(X) d(X, Y)
$$

Theorem 5. If $X$ and $Y$ are isomorphic Banach spaces, then

$$
\frac{J(X)}{d(X, Y)} \leq J(Y) \leq J(X) d(X, Y)
$$

and

$$
\frac{C_{\mathrm{NJ}}(X)}{d(X, Y)^{2}} \leq C_{\mathrm{NJ}}(Y) \leq C_{\mathrm{NJ}}(X) d(X, Y)^{2} .
$$

In particular, if $X$ and $Y$ are isometric, then $J(X)=J(Y)$ and $C_{\mathrm{NJ}}(X)=$ $C_{\mathrm{NJ}}(Y)$.

Proof. Let $x, y \in S_{X}$. For each $\varepsilon>0$ there exists an isomorphism $T$ from $X$ onto $Y$ such that $\|T\| \cdot\left\|T^{-1}\right\| \leq(1+\varepsilon) d(X, Y)$. Put

$$
x^{\prime}=\frac{T x}{\|T\|}, \quad y^{\prime}=\frac{T y}{\|T\|} .
$$

Then $x^{\prime}, y^{\prime} \in B_{Y}$ since

$$
\left\|x^{\prime}\right\|=\frac{\|T x\|}{\|T\|} \leq\|x\|=1, \quad\left\|y^{\prime}\right\|=\frac{\|T y\|}{\|T\|} \leq\|y\|=1 .
$$

By property (iv) we obtain 


$$
\begin{aligned}
& \min \{\|x+y\|,\|x-y\|\}=\|T\| \min \left\{\left\|T^{-1}\left(x^{\prime}+y^{\prime}\right)\right\|,\left\|T^{-1}\left(x^{\prime}-y^{\prime}\right)\right\|\right\} \\
& \leq(1+\varepsilon) d(X, Y) \min \left\{\left\|x^{\prime}+y^{\prime}\right\|,\left\|x^{\prime}-y^{\prime}\right\|\right\} \leq(1+\varepsilon) d(X, Y) J(Y),
\end{aligned}
$$

and since $x, y \in S_{X}$ were arbitrary, $J(X) \leq(1+\varepsilon) d(X, Y) J(Y)$, which gives the first estimate in (5.2). The second estimate follows by just interchanging $X$ and $Y$. The estimates in (5.3) can be proved similarly.

Corollary 4. Let $X=(X,\|\cdot\|)$ be a non-trivial Banach space and let $X_{1}=\left(X,\|\cdot\|_{1}\right)$, where $\|\cdot\|_{1}$ is an equivalent norm on $X$ satisfying, for $\alpha, \beta>0$ and $x \in X$,

$$
\alpha\|x\| \leq\|x\|_{1} \leq \beta\|x\| .
$$

Then $\frac{\alpha}{\beta} N(X) \leq N\left(X_{1}\right) \leq \frac{\beta}{\alpha} N(X)$ and

$$
\begin{gathered}
\frac{\alpha}{\beta} J(X) \leq J\left(X_{1}\right) \leq \frac{\beta}{\alpha} J(X), \\
\frac{\alpha^{2}}{\beta^{2}} C_{\mathrm{NJ}}(X) \leq C_{\mathrm{NJ}}\left(X_{1}\right) \leq \frac{\beta^{2}}{\alpha^{2}} C_{\mathrm{NJ}}(X) .
\end{gathered}
$$

Proof. This follows immediately from Theorem 5 and the fact that $d\left(X, X_{1}\right) \leq \beta / \alpha$.

We say that a Banach space $X$ is finitely representable in a Banach space $Y$ (James [21]; cf. Beauzamy [3], p. 217) if, for any $\varepsilon>0$ and for every finite-dimensional subspace $X_{0}$ of $X$, there exists a finite-dimensional subspace $Y_{0}$ of $Y$ with $\operatorname{dim} Y_{0}=\operatorname{dim} X_{0}$ such that $d\left(X_{0}, Y_{0}\right) \leq 1+\varepsilon$.

Corollary 5. (i) Let $X$ be a Banach space which is finitely representable in $Y$. Then $J(X) \leq J(Y), C_{\mathrm{NJ}}(X) \leq C_{\mathrm{NJ}}(Y)$ and $N(X) \leq N(Y)$.

(ii) $J\left(X^{* *}\right)=J(X), N\left(X^{* *}\right)=N(X)$.

Proof. (i) It is almost the same as the proof of Theorem 5 and (5.1) so we leave out the details.

(ii) By (i) and the principle of local reflexivity which asserts that $X^{* *}$ is finitely representable in $X$ (cf. [41]) it follows that $J\left(X^{* *}\right) \leq J(X)$. On the other hand, $X$ is isometric to a subspace $Y$ of $X^{* *}$ and, therefore, $J(X)=$ $J(Y) \leq J\left(X^{* *}\right)$. The proof of the second equality in (ii) is similar.

Remark 11. Let $X$ be an infinite-dimensional Banach space and let

$$
\begin{aligned}
& p_{X}=\sup \{p \in[1,2]: X \text { is of type } p\} \\
& q_{X}=\inf \{q \in[2, \infty]: X \text { is of cotype } q\}
\end{aligned}
$$

(see [30] for details). By a theorem of Maurey-Pisier [33], $\ell_{p_{X}}$ and $\ell_{q_{X}}$ are both finitely representable in $X$. Thus, by using Corollary $5(\mathrm{i})$ and the values of the James and Jordan-von Neumann constants for $\ell_{p}$-spaces, we obtain 
the following estimates:

$$
\begin{aligned}
J(X) & \geq \max \left\{2^{1 / p_{X}}, 2^{1-1 / q_{X}}\right\}, \\
C_{\mathrm{NJ}}(X) & \geq 2^{2 / \min \left\{p_{X}, q_{X}^{\prime}\right\}-1}, \\
N(X) & \geq \min \left\{2^{1 / p_{X}}, 2^{1-1 / q_{X}}\right\} .
\end{aligned}
$$

In particular, if $J(X)=\sqrt{2}$, then $p_{X}=q_{X}=2$.

Corollary 6. If $\operatorname{dim} X=2$, then

$$
\sqrt{2} / d\left(X, \ell_{2}^{2}\right) \leq N(X) \leq \sqrt{2} d\left(X, \ell_{2}^{2}\right), \quad \sqrt{2} / d\left(X, \ell_{2}^{2}\right) \leq J(X) \leq \sqrt{2} d\left(X, \ell_{2}^{2}\right)
$$

and

$$
1 / d\left(X, \ell_{2}^{2}\right)^{2} \leq C_{\mathrm{NJ}}(X) \leq d\left(X, \ell_{2}^{2}\right)^{2}
$$

Remark 12. By using Corollary 6 we can also prove Remark 8. In fact, if $C_{\mathrm{NJ}}(X)=2$, then since $d\left(X, \ell_{2}^{2}\right) \leq \sqrt{2}$, it follows from Corollary 6 that

$$
2=C_{\mathrm{NJ}}(X) \leq d\left(X, \ell_{2}^{2}\right)^{2} \leq 2
$$

and so $d\left(X, \ell_{2}^{2}\right)=\sqrt{2}$. Therefore a two-dimensional space $X$ is isometric to $\ell_{\infty}^{2}$ (cf. [39], Proposition 37.4) and we have in fact proved the crucial part of Remark 8.

We illustrate the above remarks and results by the following examples. They will show that evidently the James constant, the Jordan-von Neumann constant and the normal structure coefficient are not stable with respect to equivalent renorming. On the other hand, the examples will also show that Theorem 5 and Corollaries 4-6 can give both precise estimates of these constants and only rough results.

ExAmple 6 . (a) For $2 \leq p<\infty$ and $\lambda \geq 1$ let $X_{\lambda, p}$ be the space $\ell_{p}$ with the norm

$$
\|x\|_{\lambda, p}=\max \left\{\|x\|_{p}, \lambda\|x\|_{\infty}\right\}
$$

(cf. [25] and our Example 5, with $p=2$ ). Then

$$
\|x\|_{p} \leq\|x\|_{\lambda, p} \leq \lambda\|x\|_{p} \quad \text { for all } x \in \ell_{p}
$$

and, according to Corollary 4,

$$
\begin{gathered}
J\left(X_{\lambda, p}\right) \leq \min \left\{2, \lambda J\left(\ell_{p}\right)\right\}=\min \left\{2, \lambda 2^{1-1 / p}\right\}, \\
C_{\mathrm{NJ}}\left(X_{\lambda, p}\right) \leq \min \left\{2, \lambda^{2} C_{\mathrm{NJ}}\left(\ell_{p}\right)\right\}=\min \left\{2, \lambda^{2} 2^{1-2 / p}\right\}, \\
N\left(X_{\lambda, p}\right) \leq \max \left\{1, N\left(\ell_{p}\right) / \lambda\right\}=\max \left\{1,2^{1 / p} / \lambda\right\} .
\end{gathered}
$$

Taking $x=(a / \lambda, a / \lambda, 0,0, \ldots)$ and $y=(a / \lambda,-a / \lambda, 0,0, \ldots)$ with $a=$ $\min \left(1, \lambda 2^{-1 / p}\right)$ we even obtain equalities for the first two constants:

$$
J\left(X_{\lambda, p}\right)=\min \left\{2, \lambda 2^{1-1 / p}\right\}, \quad C_{\mathrm{NJ}}\left(X_{\lambda, p}\right)=\min \left\{2, \lambda^{2} 2^{1-2 / p}\right\} .
$$


If we take, as in $[1, \mathrm{p} .146]$ where the case of $p=2$ was considered, the set $A$ as the closure of $\operatorname{conv}\left(\left\{e_{n}: n \in \mathbb{N}\right\}\right)$, then $\operatorname{diam}(A)=\max \left\{2^{1 / p}, \lambda\right\}$ and $r(A)=\lambda$ and so

$$
N\left(X_{\lambda, p}\right)=\max \left\{1,2^{1 / p} / \lambda\right\} .
$$

(b) For $1 \leq p \leq 2$ and $\lambda \geq 1$ let $Y_{\lambda, p}$ be the space $L_{p}[0,1]$ with the norm

$$
\|x\|_{\lambda, p}=\max \left\{\|x\|_{p}, \lambda\|x\|_{1}\right\} .
$$

As in (a) we obtain

$$
J\left(Y_{\lambda, p}\right)=\min \left\{2, \lambda 2^{1 / p}\right\}, \quad C_{\mathrm{NJ}}\left(Y_{\lambda, p}\right)=\min \left\{2, \lambda^{2} 2^{2 / p-1}\right\}
$$

(equalities are realized for $x=a \chi_{[0,1 / 2)}$ and $y=a \chi_{[1 / 2,1]}$ with $a=$ $\left.\min \left\{2^{1 / p}, 2 / \lambda\right\}\right)$ and

$$
N\left(Y_{\lambda, p}\right) \geq \max \left\{1,2^{1-1 / p} / \lambda\right\} .
$$

Taking as $K$ the set formed by the Rademacher functions $r_{1}, \ldots, r_{m}$ we have $\left\|r_{k}\right\|_{\lambda, p}=1$ and $\left\|r_{k}-r_{j}\right\|_{\lambda, p}=\max \left\{2^{1-1 / p}, \lambda\right\}$ for all $k \neq j$ and also (cf. [1, p. 129]) $r(K) \geq(1-1 / m) \lambda$, which gives

$$
\operatorname{diam}(K) / r(K) \leq \frac{\max \left\{2^{1-1 / p}, \lambda\right\}}{(1-1 / m) \lambda} \rightarrow \max \left\{1,2^{1-1 / p} / \lambda\right\} \quad \text { as } m \rightarrow \infty .
$$

Therefore $N\left(Y_{\lambda, p}\right)=\max \left\{1,2^{1-1 / p} / \lambda\right\}$.

Example 7 . For $\lambda>0$ let $Z_{\lambda}$ be $\mathbb{R}^{2}$ with the norm

$$
|x|_{\lambda}=\left(\|x\|_{2}^{2}+\lambda\|x\|_{\infty}^{2}\right)^{1 / 2} .
$$

Then

$$
\|x\|_{2} \leq|x|_{\lambda} \leq(1+\lambda)^{1 / 2}\|x\|_{2} \quad \text { for all } x \in \mathbb{R}^{2},
$$

and the estimates we get by using Corollary $4: J\left(Z_{\lambda}\right) \leq \min \left\{2,(2+2 \lambda)^{1 / 2}\right\}$, $C_{\mathrm{NJ}}\left(Z_{\lambda}\right) \leq \min \{2,1+\lambda\}, N\left(Z_{\lambda}\right) \geq \max \left\{1,\left[(2 /(1+\lambda)]^{1 / 2}\right\}\right.$, are rather poor, because calculations show that

$$
J\left(Z_{\lambda}\right)=2[(\lambda+1) /(\lambda+2)]^{1 / 2} \text { and } C_{\mathrm{NJ}}\left(Z_{\lambda}\right)=2(\lambda+1) /(\lambda+2) .
$$

ExAmple 8 (Day-James $\ell_{p}-\ell_{q}$ spaces and Bynum $b_{p, q}$-spaces). For $1 \leq$ $p, q \leq \infty$ denote by $\ell_{p}-\ell_{q}$ the Day-James space, i.e., $\mathbb{R}^{2}$ with the norm defined by

$$
|x|_{p, q}= \begin{cases}\|x\|_{p} & \text { if } x_{1} x_{2} \geq 0 \\ \|x\|_{q} & \text { if } x_{1} x_{2} \leq 0\end{cases}
$$

James $\left[19\right.$, p. 561] considered the $\ell_{p^{-}} \ell_{p^{\prime}}$ space as an example of a Banach space which is isometric to its dual but which is not given by a Hilbert norm when $p \neq 2$. Day [10] considered even more general spaces, namely, if $(X,\|\cdot\|)$ is a two-dimensional Banach space and $\left(X^{*},\|\cdot\|^{*}\right)$ its dual, then 
the $X-X^{*}$ space is the space $X$ with the norm defined by

$$
|x|_{X, X^{*}}= \begin{cases}\|x\| & \text { if } x_{1} x_{2} \geq 0 \\ \|x\|^{*} & \text { if } x_{1} x_{2} \leq 0 .\end{cases}
$$

The infinite-dimensional version of the Day-James $\ell_{p}-\ell_{q}$ space is the space $b_{p, q}$ which was investigated by Bynum [4] (usually this space is denoted by $\ell_{p, q}$, but this notation suggests the standard Lorentz sequence spaces, therefore we use the notation $b_{p, q}$ instead). For $1 \leq p, q \leq \infty$ denote by $b_{p, q}$ the $\ell_{p}$ space with the norm

$$
|x|_{p, q}= \begin{cases}\left(\left\|x^{+}\right\|_{p}^{q}+\left\|x^{-}\right\|_{p}^{q}\right)^{1 / q} & \text { when } 1 \leq q<\infty \\ \max \left\{\left\|x^{+}\right\|_{p},\left\|x^{-}\right\|_{p}\right\} & \text { when } q=\infty\end{cases}
$$

where $x^{+}$and $x^{-}$are positive and negative parts of $x \in \ell_{p}$, i.e., $\left(x^{+}\right)_{n}=$ $\max \left\{x_{n}, 0\right\}$ and $\left(x^{-}\right)_{n}=\max \left\{-x_{n}, 0\right\}$. Note that if $p \geq q \geq 1$, then, by the convexity of the function $f(u)=u^{p / q}$, we have

$$
\|x\|_{p} \leq|x|_{p, q} \leq 2^{1 / q-1 / p}\|x\|_{p} .
$$

In view of Corollary 4 , this yields that if either $X=\ell_{p}-\ell_{q}$ or $X=b_{p, q}$, then $J(X) \leq \min \left\{2,2^{1 / q-1 / p} J\left(\ell_{p}\right)\right\}, C_{\mathrm{NJ}}(X) \leq \min \left\{2,2^{2 / q-2 / p} C_{\mathrm{NJ}}\left(\ell_{p}\right)\right\}$ and $N(X) \geq \max \left\{1,2^{1 / p-1 / q} N\left(\ell_{p}\right)\right\}$. From these rough estimates we cannot get the following information (cf. our Examples 2 and 4$)$ :

$$
J\left(\ell_{2}-\ell_{1}\right)=\sqrt{8 / 3}, \quad C_{\mathrm{NJ}}\left(\ell_{2}-\ell_{1}\right) \geq 3 / 2
$$

and

$$
J\left(\ell_{\infty}-\ell_{1}\right)=3 / 2, \quad C_{\mathrm{NJ}}\left(\ell_{\infty}-\ell_{1}\right) \geq 5 / 4 .
$$

Problem 1 (cf. [12]). Let $1<p<\infty, 1 \leq q<\infty$. Compute $N\left(b_{p, q}\right)$.

Problem 2. Compute $J(X)$ and $C_{\mathrm{NJ}}(X)$ for $X=\ell_{p}-\ell_{q}$ or $X=b_{p, q}$.

Acknowledgements. This research started when the second named author visited the Department of Mathematics, Kyushu Institute of Technology (KIT), Kitakyushu, Japan, in November-December 1996 and JanuaryMarch 1997. He was then supported by KIT and partly by the Swedish Natural Science Research Council (NFR). At the second visit in 1997 the work was "almost" finished and the first named author presented the results (Theorems 1, 3 and 4) at the conferences in Japan (Kyoto, Aug. 27, 1997-see published results without proofs in the conference proceedings [24]), Poland (Poznań, Aug. 30, 1998) and on seminars in Sweden (Luleå, Sept. 9, 1998) and Spain (Madrid, Sept. 22, 1999). The paper was completed by examples and finally finished when the second named author visited KIT in January-March 2000. This time he was supported by the Japan Society for the Promotion of Science (JSPS; grant S-99189). He is deeply indebted to the Department of Mathematics of KIT for their kind hospitality during all his stays and to JSPS for their financial support. The research of 
the first and third named authors was also supported by the Japan Society for the Promotion of Science. The authors also thank Professor S. Prus for providing Example 2 and Professor L. E. Persson for reading through and commenting our paper.

\section{References}

[1] A. Ayerbe Toledano, T. Domínguez Benavides and G. López Acedo, Measures of Noncompactness in Metric Fixed Point Theory, Oper. Theory Adv. Appl. 99, Birkhäuser, Basel, 1997.

[2] J. B. Baillon and R. Schoneberg, Asymptotic normal structure and fixed points of nonexpansive mappings, Proc. Amer. Math. Soc. 81 (1981), 257-264.

[3] B. Beauzamy, Introduction to Banach Spaces and their Geometry, 2nd ed., NorthHolland, Amsterdam, 1985.

[4] W. L. Bynum, A class of spaces lacking normal structure, Compositio Math. 25 (1972), 233-236.

[5] -, Normal structure coefficients for Banach spaces, Pacific J. Math. 86 (1980), 427-436.

[6] E. Casini, About some parameters of normed linear spaces, Atti. Acad. Naz. Lincei Rend. Cl. Sci. Fis. Mat. Natur. (8) 80 (1986), 11-15.

[7] J. A. Clarkson, Uniformly convex spaces, Trans. Amer. Math. Soc. 40 (1936), 396414.

[8] -, The von Neumann-Jordan constant for the Lebesgue spaces, Ann. of Math. 38 (1937), 114-115.

[9] M. M. Day, Some characterizations of inner product spaces, Trans. Amer. Math. Soc. 62 (1947), 320-337.

[10] - Uniform convexity in factor and conjugate spaces, Ann. of Math. 45 (1944), 375-385.

[11] T. Domínguez Benavides, Normal structure coefficients of $L^{p}(\Omega)$, Proc. Roy. Soc. Edinburgh Sect. A 117 (1991), 299-303.

[12] T. Domínguez Benavides, G. López Acedo and H.-K. Xu, Qualitative and quantitative properties for the space $\ell_{p, q}$, Houston J. Math. 22 (1996), 89-100.

[13] T. Figiel, On the moduli of convexity and smoothness, Studia Math. 56 (1976), 121-155.

[14] S. Fitzpatrick and B. Reznick, Skewness in Banach spaces, Trans. Amer. Math. Soc. 275 (1983), 587-597.

[15] J. Gao and K. S. Lau, On the geometry of spheres in normed linear spaces, J. Austral. Math. Soc. Ser. A 48 (1990), 101-112.

[16] - - - On two classes of Banach spaces with uniform normal structure, Studia Math. 99 (1991), 41-56.

[17] K. Goebel and W. A. Kirk, Topics in Metric Fixed Point Theory, Cambridge Univ. Press, 1990.

[18] N. M. Gulevich, Fixed points of nonexpansive mappings, J. Math. Sci. 79 (1996), 755-815.

[19] R. C. James, Inner products in normed linear spaces, Bull. Amer. Math. Soc. 53 (1947), 559-566. 
[20] R. C. James, Uniformly non-square Banach spaces, Ann. of Math. 80 (1964), 542550 .

[21] —, Some self-dual properties of normed linear spaces, in: Sympos. Infinite Dim. Topology (Baton Rouge, 1967), Ann. of Math. Stud. 69, Princeton Univ. Press, 1972, 159-175.

[22] A. Jiménez-Melado and E. Llorens-Fuster, The fixed point property for some uniformly nonsquare Banach spaces, Boll. Un. Mat. Ital. A 10 (1996), 587-595.

[23] P. Jordan and J. von Neumann, On inner products in linear metric spaces, Ann. of Math. 36 (1935), 719-723.

[24] M. Kato, L. Maligranda and Y. Takahashi, Von Neumann-Jordan constant and some geometrical constants of Banach spaces, in: Nonlinear Analysis and Convex Analysis, Res. Inst. Math. Sci. 1031, Kyoto Univ., Kyoto, 1998, 68-74.

[25] M. Kato and Y. Takahashi, On the von Neumann-Jordan constant for Banach spaces, Proc. Amer. Math. Soc. 125 (1997), 1055-1062.

[26] -, - Von Neumann-Jordan constant for Lebesgue-Bochner spaces, J. Inequal. Appl. 2 (1998), 89-97.

[27] M. A. Khamsi, Uniform smoothness implies super-normal structure property, Nonlinear Anal. 19 (1992), 1063-1069.

[28] J. Lindenstrauss, On the modulus of smoothness and divergent series in Banach spaces, Michigan Math. J. 10 (1963), 241-252.

[29] J. Lindenstrauss and L. Tzafriri, Classical Banach Spaces I. Sequence Spaces, Springer, Berlin, 1977.

[30] —, - Classical Banach Spaces II. Function Spaces, Springer, Berlin, 1979.

[31] L. Maligranda, Orlicz Spaces and Interpolation, Seminars in Math. 5, Univ. Estadual de Campinas, Campinas SP, 1989.

[32] E. Maluta, Uniformly normal structure and related coefficients, Pacific J. Math. 111 (1984), 357-369.

[33] B. Maurey et G. Pisier, Séries de variables aléatoires vectorielles indépendantes et propriétés géométriques des espaces de Banach, Studia Math. 58 (1976), 45-90.

[34] R. E. Megginson, An Introduction to Banach Space Theory, Grad. Texts in Math. 183, Springer, New York, 1998.

[35] V. D. Milman, Geometric theory of Banach spaces. II. Geometry of the unit sphere, Uspekhi Mat. Nauk 26 (1971), no. 6, 73-149 (in Russian); English transl.: Russian Math. Surveys 26 (1971), no. 6, 79-163.

[36] S. Prus, Some estimates for the normal structure coefficient in Banach spaces, Rend. Circ. Mat. Palermo 40 (1991), 128-135.

[37] M. A. Smith and B. Turett, Some examples concerning normal and uniform normal structure in Banach spaces, J. Austral. Math. Soc. Ser. A 48 (1990), 223-234.

[38] Y. Takahashi and M. Kato, Von Neumann-Jordan constant and uniformly nonsquare Banach spaces, Nihonkai Math. J. 9 (1998), 155-169.

[39] N. Tomczak-Jaegermann, Banach-Mazur Distances and Finite-Dimensional Operator Ideals, Pitman Monographs Surveys Pure Appl. Math. 38, Longman Sci. \& Tech. and Wiley, New York, 1989.

[40] B. Turett, A dual view of a theorem of Baillon, in: Nonlinear Analysis and Applications, Lecture Notes in Pure and Appl. Math. 80, Dekker, 1982, 279-286. 
[41] P. Wojtaszczyk, Banach Spaces for Analysts, Cambridge Univ. Press, Cambridge, 1991.

Department of Mathematics

Kyushu Institute of Technology

Tobata, Kitakyushu 804-8550, Japan

E-mail: katom@tobata.isc.kyutech.ac.jp

Department of System Engineering

Okayama Prefectural University

Soja 719-1197, Japan

E-mail: takahasi@cse.oka-pu.ac.jp
Department of Mathematics Luleå University of Technology S-971 87 Luleå, Sweden E-mail: lech@sm.luth.se

Received March 27, 2000

Revised version August 31, 2000 